\title{
Ameliorating process parameters for zeaxanthin yield in Arthrobacter gandavensis MTCC 25325
}

\section{CURRENT STATUS: UNDER REVISION}

AMB Express $\underline{\text { Springer }}$

\section{Shristi Ram}

Central Salt and Marine Chemicals Research Institute- CSIR

Sushma Rani Tirkey

Central Salt and Marine Chemicals Research Institute CSIR

Madhava Anil Kumar

Central Salt and Marine Chemicals Research Institute CSIR

Sandhya Mishra

CSIR-Central Salt and Marine Chemicals Research Institute

smishra@csmcri.orgCorresponding Author

\section{DOI:}

10.21203/rs.2.22112/v1

\section{SUBJECT AREAS}

General Microbiology

\section{KEYWORDS}

Zeaxanthin, Arthrobacter gandavensis, Central Composite Design, Nutraceutical 
Abstract

The present study aims to screen potential bacterial isolates for the production of a prophylactic agent zeaxanthin; which is considered to be an inevitable inclusion in human diet. A freshwater bacterium Arthrobacter gandavensis was isolated from Bor Talav, Bhavnagar and screened as a promising producer of zeaxanthin. The chemo-metric tools were employed to optimize the influencing factors such as $\mathrm{pH}$, temperature, inoculum size, agitation speed, carbon source and harvest time on its yield. Thereafter, 6 parameters were narrowed down to 3 factors and were optimized using the central composite design (CCD) matrix. Maximum zeaxanthin $(1.51 \mathrm{mg} / \mathrm{g})$ was derived when $A$. gandavensis was grown under $\mathrm{pH} 6.0,1.5 \%(\mathrm{w} / \mathrm{v})$ glucose and 10\% (v/v) inoculum size. A high regression coefficient ( $R 2=0.92$ ) of the developed model indicated the accurateness with the tested parameters. To the best of our knowledge, this is the first report on tailoring of process parameters using chemo-metric optimization for escalating the zeaxanthin production by $A$. gandavensis.

\section{Full Text}

Due to technical limitations, full-text HTML conversion of this manuscript could not be completed. However, the manuscript can be downloaded and accessed as a PDF.

\section{Tables}

Table 1 Development of various zone of inhibition $(\mathrm{mm})$ exerted by the bacterial isolates on different antibiotics

'-' indicates resistant

Table 2 CCD matrix showing the zeaxanthin yield on third day, agitation at $120 \mathrm{rpm}$ and $40^{\circ} \mathrm{C}$ using $A$. gandavensis 


\begin{tabular}{|c|c|c|c|c|c|c|}
\hline Antibiotics & $\begin{array}{l}\text { Planomicrobium } \\
\text { okeanokoites }\end{array}$ & $\begin{array}{l}\text { Arthrobacter } \\
\text { gandavensis }\end{array}$ & $\begin{array}{l}\text { Kocuria } \\
\text { flava }\end{array}$ & $\begin{array}{l}\text { Rhodococcus } \\
\text { ruber }\end{array}$ & $\begin{array}{l}\text { Planococcu } \\
\text { s maritimus }\end{array}$ & $\begin{array}{l}\text { Kocuria } \\
\text { sp. }\end{array}$ \\
\hline Ampicillin & - & 29 & 40 & 13 & 18 & 23 \\
\hline Clarithromycin & 12 & 33 & 22 & - & 10 & 16 \\
\hline Gentamicin & 33 & 25 & 14 & 23 & 21 & 33 \\
\hline Amoxyclav & - & 23 & 31 & 19 & 19 & 30 \\
\hline Vancomycin & - & 26 & 19 & 17 & 17 & 26 \\
\hline Cephalothin & - & 24 & 40 & 36 & 34 & 40 \\
\hline Amikacin & 28 & 21 & 14 & 22 & 18 & 33 \\
\hline Novobiocin & - & 31 & 34 & 30 & 34 & 40 \\
\hline Erythromycin & 10 & 32 & 34 & - & - & 10 \\
\hline Teicoplanin & - & 24 & 25 & 16 & 16 & 24 \\
\hline Co-Trimoxazole & 21 & 30 & 28 & 10 & - & 22 \\
\hline Penicillin & - & 33 & 40 & 18 & 15 & 25 \\
\hline Azithromycin & 32 & 38 & 37 & - & 22 & 10 \\
\hline Oflaxacin & 20 & 20 & 20 & 19 & 24 & 28 \\
\hline Methicillin & - & 26 & 33 & 37 & 33 & 37 \\
\hline Linezolid & - & 37 & 31 & 24 & 25 & 32 \\
\hline Clindamycin & - & 38 & 40 & 10 & - & 20 \\
\hline Tetracycline & 20 & 28 & 32 & 11 & 15 & 40 \\
\hline Chloramphenicol & 10 & 37 & 33 & 20 & 18 & 25 \\
\hline Oxacillin & - & 16 & 23 & 26 & 32 & 39 \\
\hline
\end{tabular}




\begin{tabular}{|c|c|c|c|c|}
\hline Run & $\mathrm{pH}$ & Inoculum (\%) & Glucose (\%) & Zeaxanthin $(\mathrm{mg} / \mathrm{g})$ \\
\hline 1 & 5.0 & 10 & 1.0 & 0.07 \\
\hline 2 & 7.0 & 10 & 1.0 & 0.22 \\
\hline 3 & 5.0 & 12 & 1.0 & 0.06 \\
\hline 4 & 7.0 & 12 & 1.0 & 1.07 \\
\hline 5 & 5.0 & 10 & 2.0 & 0.12 \\
\hline 6 & 7.0 & 10 & 2.0 & 0.52 \\
\hline 7 & 5.0 & 12 & 2.0 & 0.02 \\
\hline 8 & 7.0 & 12 & 2.0 & 0.93 \\
\hline 9 & 5.0 & 11 & 1.5 & 0.00 \\
\hline 10 & 7.0 & 11 & 1.5 & 0.12 \\
\hline 11 & 6.0 & 10 & 1.5 & 1.51 \\
\hline 12 & 6.0 & 12 & 1.5 & 1.36 \\
\hline 13 & 6.0 & 11 & 1.0 & 0.92 \\
\hline 14 & 6.0 & 11 & 2.0 & 1.13 \\
\hline 15 & 6.0 & 11 & 1.5 & 0.83 \\
\hline 16 & 6.0 & 11 & 1.5 & 0.83 \\
\hline 17 & 6.0 & 11 & 1.5 & 0.83 \\
\hline 18 & 6.0 & 11 & 1.5 & 0.83 \\
\hline 19 & 6.0 & 11 & 1.5 & 0.83 \\
\hline 20 & 6.0 & 11 & 1.5 & 0.83 \\
\hline
\end{tabular}

Table 3 ANOVA for response surface polynomial equation and corresponding F-values and $p$-values for Zeaxanthin production by $A$. gandavensis

Figures 


\begin{tabular}{|c|c|c|c|c|}
\hline Source & Degree of Freedom & Adj SS & Adj MS & $\mathrm{F}-\mathrm{Va}$ \\
\hline Model & 9 & 3.95944 & 0.43994 & 14.4 \\
\hline Linear & 3 & 0.78525 & 0.26175 & 8.6 \\
\hline$x_{1}$ & 1 & 0.67081 & 0.67081 & 22.1 \\
\hline$x_{2}$ & 1 & 0.10000 & 0.10000 & 3.2 \\
\hline$x_{3}$ & 1 & 0.01444 & 0.01444 & 0.4 \\
\hline Square & 3 & 2.90165 & 0.96722 & 31. \\
\hline$x_{1}^{2}$ & 1 & 2.46055 & 2.46055 & $80 . \varepsilon$ \\
\hline$x_{2}^{2}$ & 1 & 0.50633 & 0.50633 & 16.6 \\
\hline$x_{3}{ }^{2}$ & 1 & 0.00100 & 0.00100 & 0.0 \\
\hline 2-Way Interaction & 3 & 0.27254 & 0.09085 & 2.9 \\
\hline$x_{1} x_{2}$ & 1 & 0.23461 & 0.23461 & 7.7 \\
\hline$x_{1} x_{3}$ & 1 & 0.00281 & 0.00281 & 0.0 \\
\hline$x_{2} x_{3}$ & 1 & 0.03511 & 0.03511 & 1.1 \\
\hline Error & 10 & 0.30422 & 0.03042 & \\
\hline Lack-of-fit & 5 & 0.30422 & 0.06084 & $*$ \\
\hline Pure Error & 5 & 0.00000 & 0.00000 & \\
\hline Total & 19 & 4.26365 & & \\
\hline$R^{2}$ & 0.928 & & & \\
\hline
\end{tabular}




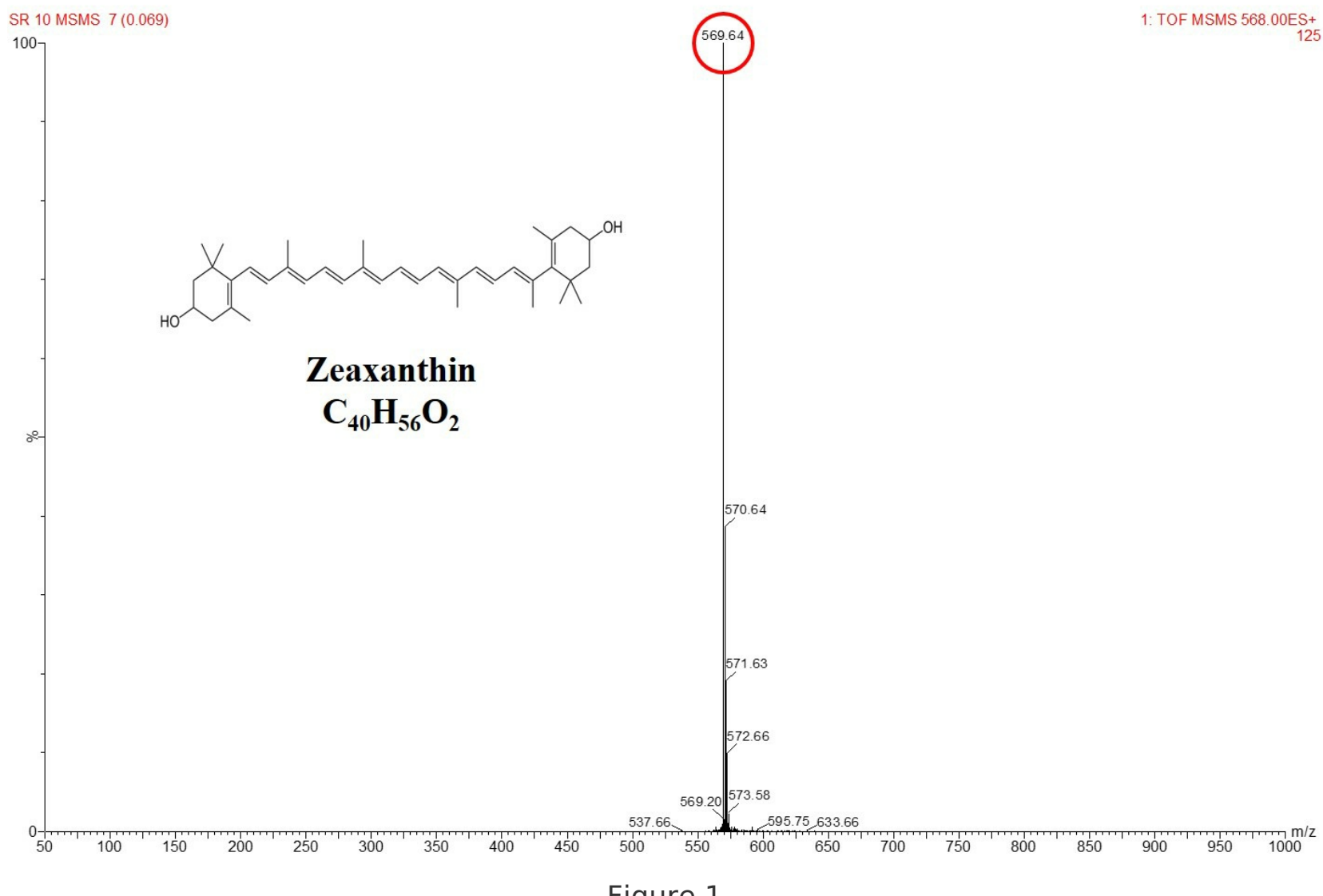

Figure 1

Graphs showing characteristic peaks of Zeaxanthin ESI-LCMS (MS/MS) in methanolic extract of A. gandavensis

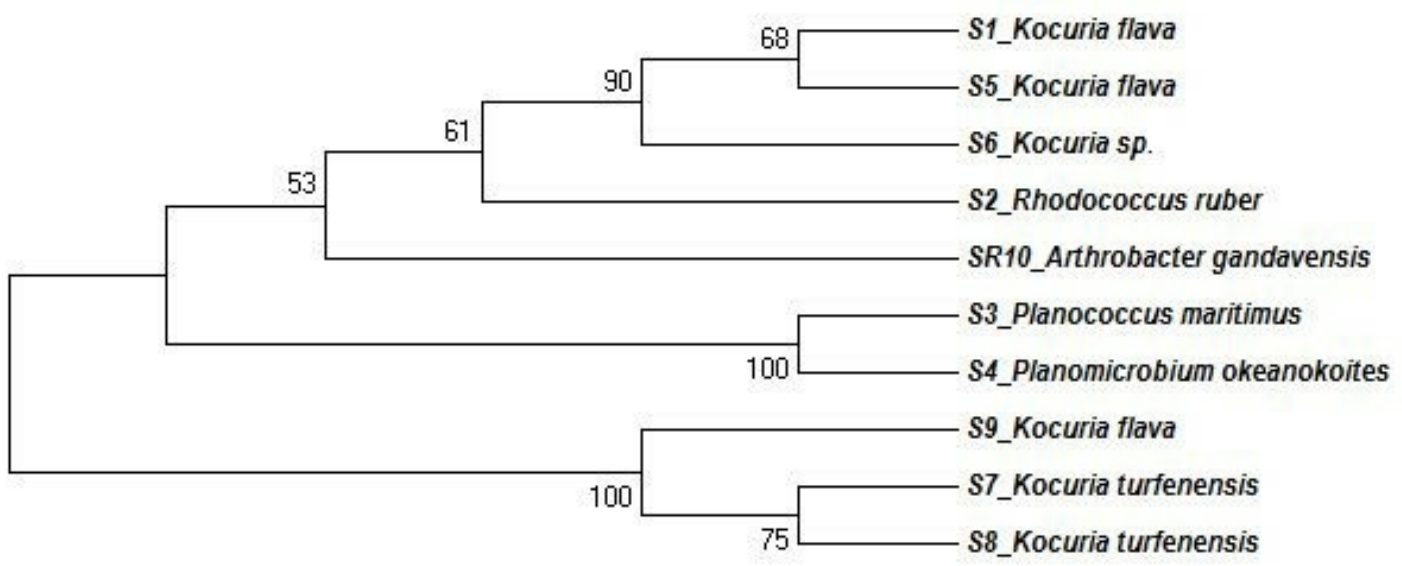

Figure 2

Phylogenetic tree of identified strains positive for zeaxanthin productions 

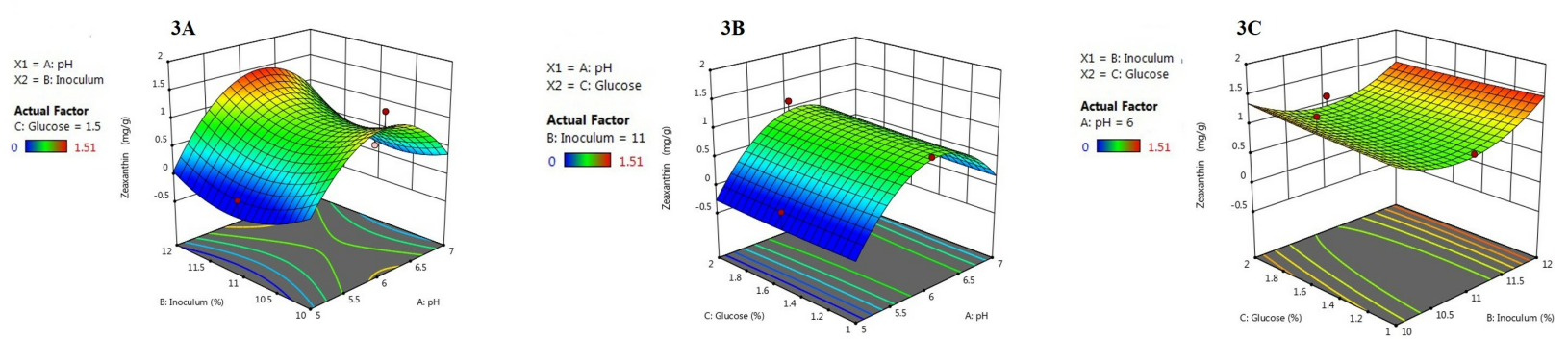

Figure 3

(a) Response surface plot of zeaxanthin Yield vs Inoculum, $\mathrm{pH}$. The regions of surface plot are divided in 3 regions depicting zeaxanthin yield in $\mathrm{mg} / \mathrm{g}$, Glucose concentration, and inoculum size in percentages. (b) Yield vs Glucose, Inoculum, and (c) Yield vs Glucose, pH.

The values are presented as mean values $\pm S D, n=3$

\section{Supplementary Files}

This is a list of supplementary files associated with this preprint. Click to download.

Supplementary Table and Figure.pdf 